\title{
Les biomarqueurs de la peroxydation lipidique
}

\section{GUICHARDANT \\ S. BACOT \\ P. MOLIÈRE \\ M. LAGARDE}

UMR 585 Inserm/Insa de Lyon Laboratoire de physiopathologie des lipides et membranes Bât. Louis Pasteur 69621 Villeurbanne, France

Fax : 0472438524

<michel.guichardant@insa-lyon.fr>

\begin{abstract}
Different biomarkers related to lipid peroxidation are available to assess an oxidative stress. They are more or less specific and sensitive. For instance malondialdehyde (MDA) or conjugated diene are easily measured in different matrix. Their measurements provide only a rough indication assessing an oxidative stress. But sometimes information is wrong due to artefacts. Some markers such as isoprostanes are specific of arachidonic acid only, and they require expensive equipment such as GC-MS/MS. They are mainly dedicated to clinical investigation. We present here new markers of oxidative stress which allow to discriminate between the oxidation of polyunsaturated fatty acid families. They are 4-hydroxynonenal (4-HNE) and 4-hydroxy-hexenal (4-HHE) which represent the peroxidation of the $n-6$ and n-3 polyunsaturated fatty acids, respectively and 4-hydroxy-dodecadienal (4-HDDE) which derives from the breakdown of 12-HpETE and mainly reflects the 12 lipoxygenation of arachidonic acid. Those markers can be easily measured with a high sensitivity by GC-MS in plasma. Their urinary carboxylic oxidized end-products, namely 4-HNA, 4-HHA and 4-HDDA may also be measured by GC-MS. The choice between these different biomarkers is not easy. It depends on which information is required.
\end{abstract}

Key words: biomakers, lipid peroxidation, oxidative stress

pour former un hydroperoxyde d'acide gras et un autre radical alkyle.

Les hydroperoxydes formés sont toxiques pour les cellules. Ils sont normalement réduits par une glutathion peroxydase en acides gras monohydroxylés. Cependant, l'activité de cette enzyme est diminuée dans le vieillissement et dans le diabète par exemple [1, 2]. II en résulte un accroissement de la durée de vie de ces hydroperoxydes qui sont instables et se dégradent en de nombreux produits tels que les alcanes, les aldéhydes, etc.

\section{Les différents marqueurs de la peroxydation lipidique}

Ils existent de nombreux marqueurs de la peroxydation lipidique. tase, glutathion peroxydase

ii) les vitamines $E$ et $C$;

iii) divers antioxydants : caroténoïdes (bêtacarotène, lycopène, etc.), les polyphénols...

La peroxydation lipidique est une conséquence du stress oxydant et aussi un relais pour sa propagation. Le mécanisme requiert un acide gras polyinsaturé avec au moins un motif 1,4cis-pentadiène. Ainsi l'acide arachidonique ( $20: 4 n-6)$ représenté à la figure 1 comporte trois de ces structures dont une est représentée en gris.

La première étape appelée phase d'initiation consiste en l'abstraction d'un radical hydrogène porté par un carbone bisallylique appartenant à cette structure 1,4-cis-pentadiène. Elle conduit à la formation d'un radical alkyle L : Les étapes ultérieures sont des étapes de propagation. Ainsi, le radical alkyle peut réagir avec un autre acide gras polyinsaturé LH pour donner un autre radical alkyle qui se réarrange en formant un diène conjugué et un autre radical alkyle $L$ : L'attaque de ce radical par une molécule d'oxygène conduit à la formation d'un radical peroxyle LOO . Ce dernier va à son tour réagir avec un autre acide gras polyinsaturé

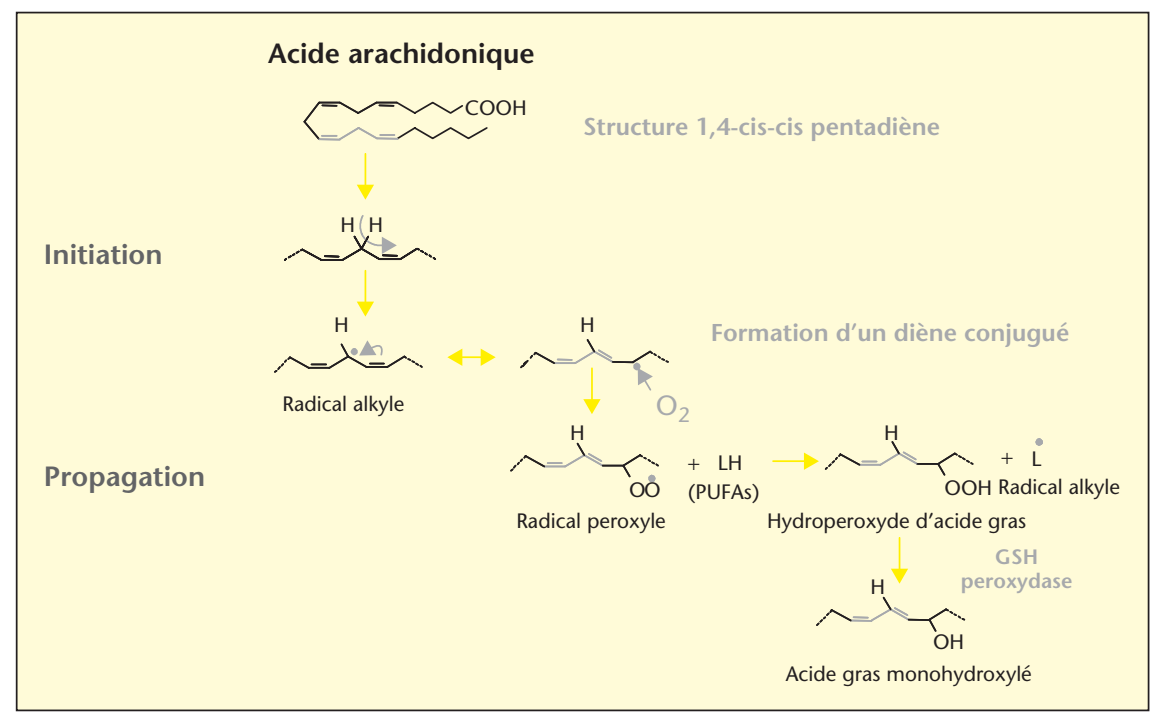

Figure 1. Schéma décrivant le mécanisme de peroxydation de l'acide arachidonique. 


\section{Les diènes conjugués}

Ils sont facilement mesurables à $\lambda 234 \mathrm{~nm}$. Leur détection, peu spécifique et peu sensible, a surtout une valeur indicative mais présente l'avantage d'une détermination cinétique.

\section{Le dialdéhyde malonique (MDA)}

C'est sans doute le plus connu et celui qui a été le plus utilisé. II résulte de la dégradation des hydroperoxydes formés au cours de la peroxydation des acides gras polyinsaturés. Cependant, il peut aussi être formé lors de l'activation de la voie de la cyclo-oxygénase. De plus, sa formation n'est pas spécifique des lipides. II peut aussi apparaître lors de l'interaction du radical hydroxyle avec la vitamine $\mathrm{C}$ ou avec le désoxyglucose. II peut être mesuré directement par GC-MS mais plus généralement détecté après sa dérivation sous la forme d'un complexe avec l'acide thiobarbiturique ou avec l'acide diéthylthiobarbiturique. Ce complexe est séparé par HPLC et détecté en UV ou par fluorimétrie. On peut le mesurer aussi bien dans les fluides (urine, sérum) que dans les cellules ou les tissus. A titre d'exemple, la figure 2 montre un profil HPLC du complexe MDA-DETBA isolé à partir d'urine.

La mesure des complexes MDA-TBA ou MDADETBA est facile à mettre en œuvre. Elle est relativement sensible, notamment si on utilise la détection fluorimétrique. Elle prend en compte la peroxydation des acides gras polyinsaturés $n-3$ et $n-6$ possédant plus de deux doubles liaisons mais elle manque de spécificité à cause des multiples sources de MDA.

\section{Les isoprostanes}

Les isoprostanes $F_{2}$ appartiennent à la famille des eicosanoïdes. Ils résultent de l'oxydation de l'acide arachidonique et sont constitués de cinq types d'isoprostanes regroupant chacun huit régioisomères [4] (figure 3). Le 8-épi-PGF 20 est l'isomère majoritaire. II peut être mesuré notamment dans les urines et est considéré actuellement comme le marqueur de référence de la peroxydation lipidique in vivo [5-8].

Pour être fiables ces marqueurs doivent être mesurés par chromatographie en phase gazeuse couplée à la spectrométrie de masse en tandem [9]. Pour cela ils doivent être dérivés sous forme de pentafluorobenzyl ester, et de triméthylsilyl ether, comme cela est décrit dans la figure 4.

Ainsi, ils peuvent être détectés avec une très grande sensibilité (de l'ordre du pg) en utilisant l'ionisation chimique négative.

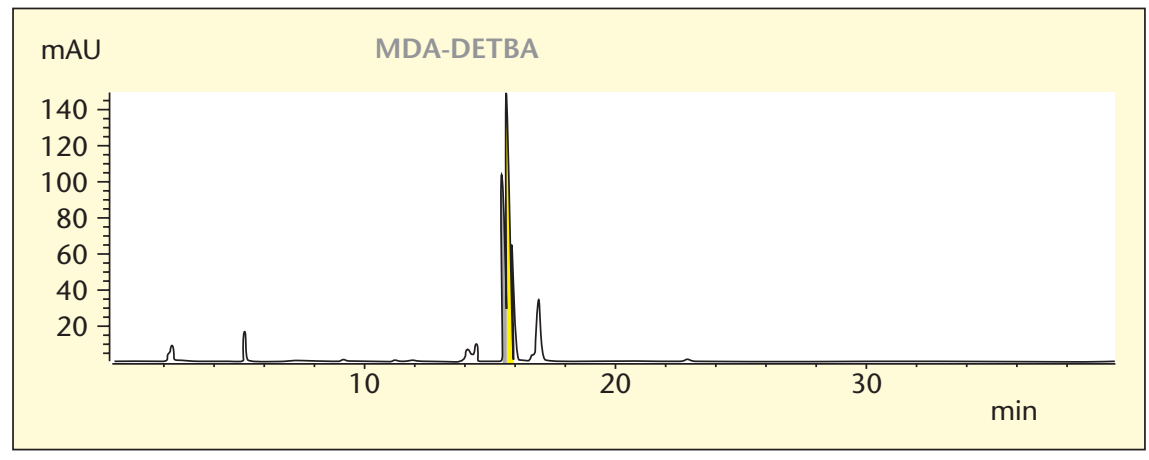

Figure 2. Profil HPLC typique du complexe MDA-DETBA. Détection UV à 539 nm; colonne Nova-Pak C18 de 30 $\mathrm{cm} \times 3,9 \mathrm{~mm}$ remplie avec des particule de $4 \mu \mathrm{m}$. Élution avec un gradient; le solvant initial est un mélange acétonitrile/eau $(10: 90 ; \mathrm{V} / \mathrm{V})$ contenant 0,1\% de triéthanolamine, puis I'acétonitrile est augmentée jusqu'à $60 \%$ à $20 \mathrm{~min}$ avant un retour aux conditions initiales à $30 \mathrm{~min}$.

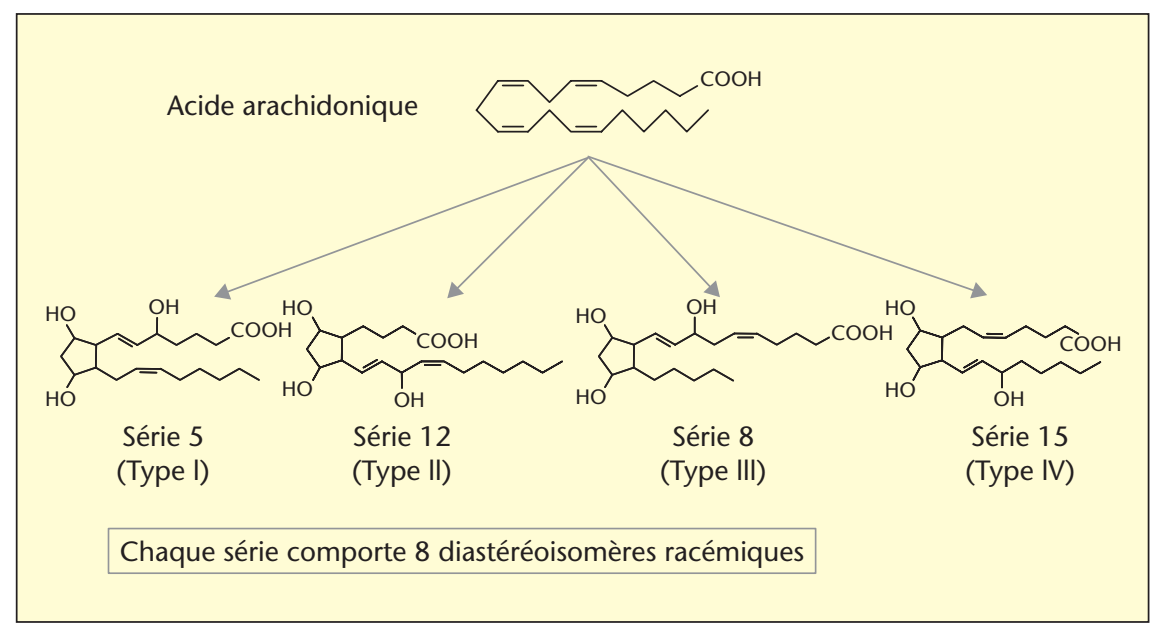

Figure 3. Génération des différentes familles d'isoprostanes (d'après Morrow JD et al. Anal Biochem 1990; 184 : 1-10)



Figure 4. Structure du 8-épi-PGF $F_{2 \alpha}$ dérivé sous forme de pentafluorobenzyl ester et de triméthylsilyl ether. 
Compte tenu du grand nombre $d^{\prime}$ isomères présents, la mesure des isoprostanes n'est pas très facile (figure 5, [9]) et nécessite, pour être fiable, un équipement GC-MS-MS. Ces marqueurs ne prennent en compte que la peroxydation de l'acide arachidonique. Cependant, il est possible de mesurer de la même manière les neuroprostanes qui dérivent de l'acide docosahexaénoïque (22:6n-3) [10,11].

\section{Les 4-hydroxy-alkénals}

Ils dérivent comme pour le MDA de la scission des hydroperoxydes formés au cours de la peroxydation lipidique des PUFA selon le schéma présenté dans la figure 6.

Ces hydroxyl-alkénals présentés dans la figure 7 ont en commun une double liaison entre les carbones 2 et 3 . Ils sont caractérisés par la présence d'un groupement aldéhydique porté par le carbone 1 et par un groupement hydroxyle sur le carbone 2 . Ils ne diffèrent que par la longueur de leur chaîne carbonée et par la présence d'une double liaison supplémentaire pour le 4-HDDE.

Ils peuvent être facilement mesurés par GC-MS après dérivation sous forme de pentafluorobenzyl oxime et de triméthylsilyl éther [12] (figure 8).

\section{Les acides urinaires dérivés des 4-hydroxyl-alkénals}

Les 4-hydroxy-alkénals sont métabolisés au niveau du foie en différents métabolites et notamment chez l'homme en acides selon le schéma présenté dans la figure 9.

Ces acides urinaires peuvent être mesurés par GC-MS sous forme de dérivés pentafluorobenzyl et heptafluorobenzyl esters.

Un exemple de séparation de ces marqueurs est donné figure 10.

Des résultats préliminaires montrent par exemple que l'ensemble de ces marqueurs sont augmentés dans les urines de patients atteints de mucovisidose alors que seul le 4-HDDA est augmenté dans le diabète [13] où une diminution de l'activité des glutathion peroxydases plaquettaires a été rapportée [2].

Ainsi, il est difficile de choisir le bon marqueur. Certains sont complémentaires, ils sont plus ou moins spécifiques et plus ou moins faciles à mettre en œuvre [3].



Figure 5. Exemple de séparation par GC-MS/MS d'isoprostanes dérivés sous forme de pentafluorobenzyl ester et de triméthylsilyl éther (d'après Anal Biochem 1999; 272 : 209-12).

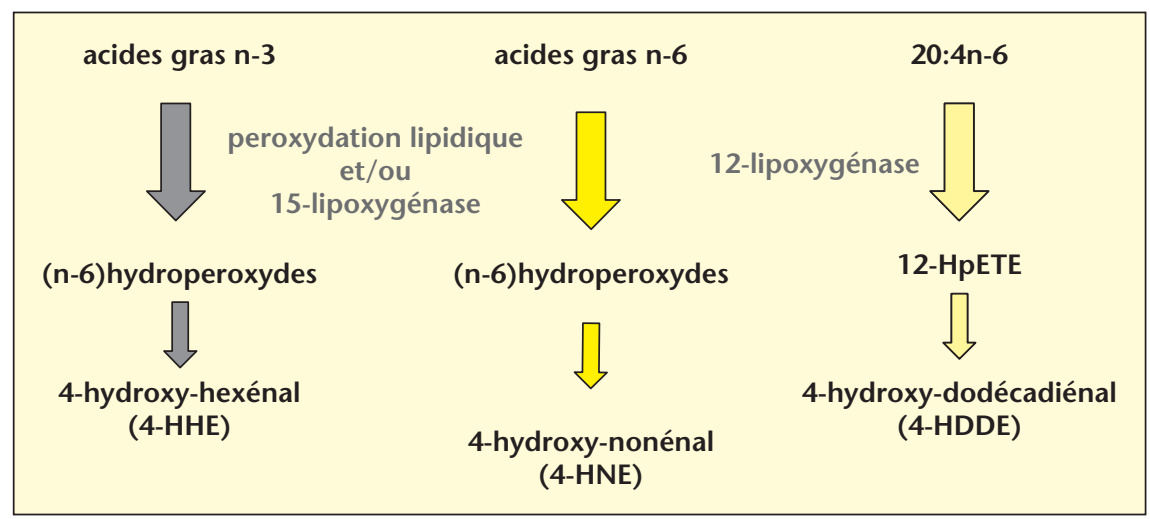

Figure 6. Schéma montrant les mécanismes conduisant à la formation des hydroxy-alkénals à partir de la peroxydation lipidique des acides gras polyinsaturés des séries $n-6$ et $n-3$ ainsi qu'à partir de l'acide arachidonique via l'activation de l'enzyme 12-lypoxygénase.

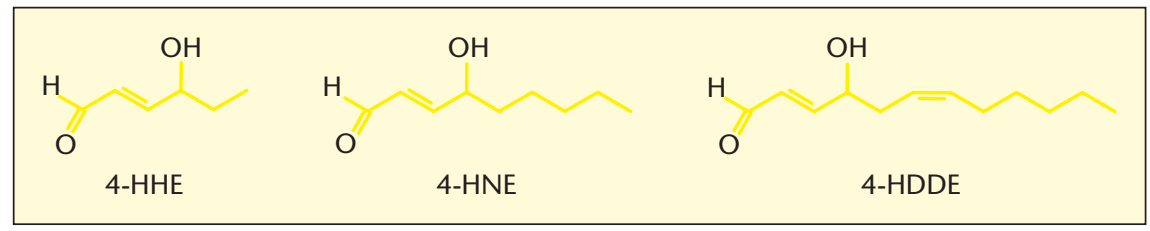

Figure 7. Structure des différents hydroxy-alkénals : 4-HHE, 4-HNE et 4-HDDE.

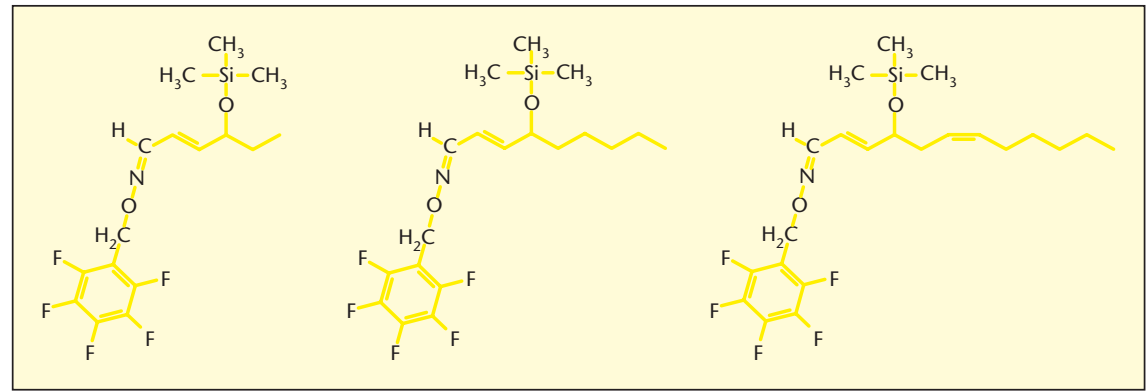

Figure 8. Structure des hydroxy-alkénals dérivés sous forme de pentafluorobenzyl oxime et de triméthylsilyl éther. 


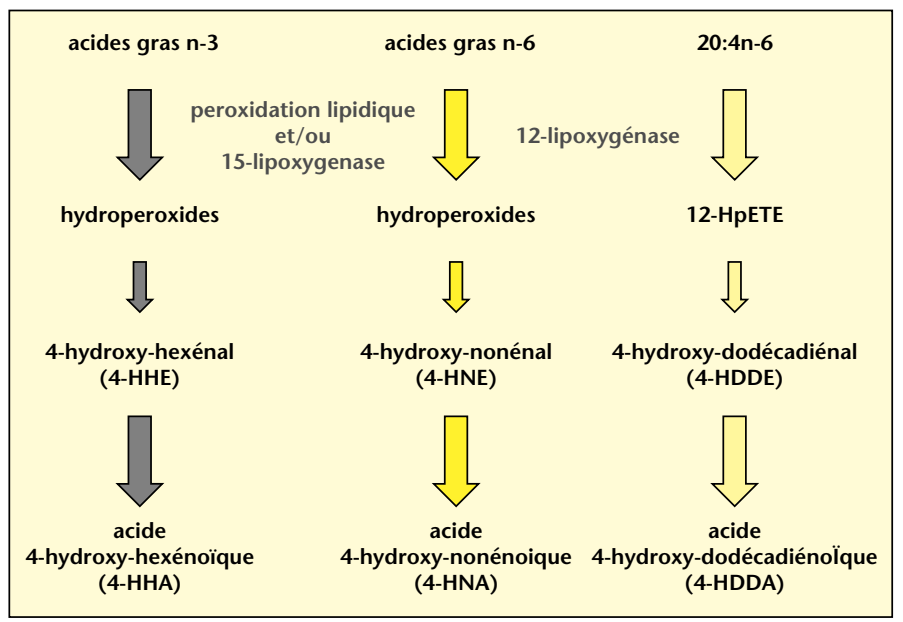

Figure 9. Schéma montrant la formation des acides 4-HHA, 4-HNA et 4-HDDA par oxydation des hydroxy-alkénals correspondants.

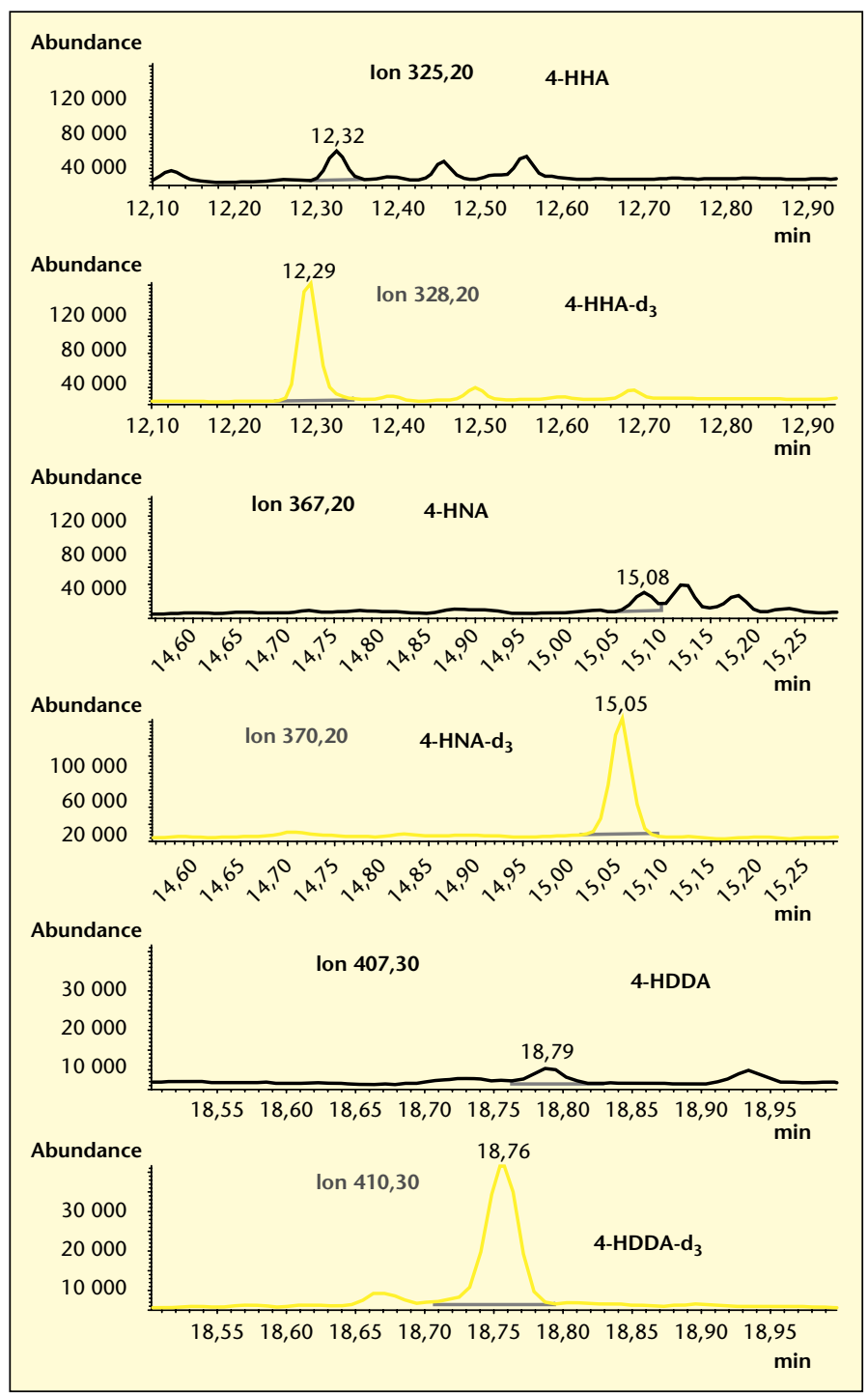

Figure 10. Exemple de séparation des acides 4-HHA, 4-HNA, 4-HDDA extraits d'urine d'un diabétique et dérivés sous forme de pentafluorobenzyl ester et d'heptafluorobutyryl ester et analysés par GC-MS en mode NICI. Les molécules deutérées servent de standards internes.

\section{RÉFÉRENCES}

1. MURUGANANDAM A, DROUILLARD C, THIBERT R, CHEUNG R, DRAISEY T, MUTUS B. Glutathione metabolic enzyme activities in diabetic platelets as a function of glycemic control. Thromb Res $1992 ; 67$ : 385-97.

2. VERICEL E, JANUEL C, CARRERAS M, MOULIN $P$, LAGARDE M. Diabetic patients without vascular complications display enhanced basal platelet activation and decrease antioxidant status. Diabetes $2004 ; 53: 1046-51$.

3. GUICHARDANT M, VALETTE-TALBI L, CAVADINI C, CROZIER G, BERGER M. Malondialdehyde measurement in urine. I Chromatogr 1994 ; 655 : 112-6.

4. MORROW JD, HARRIS TM, ROBERTS 2ND LI. Non cyclooxygenase oxidative formation of a series of novel prostaglandins : Analytical ramifications for measurement of eicosanoids. Anal Biochem $1990 ; 181$ : 1-10.

5. ROBERTS II LJ, MORROW JD. Measurement of $\mathrm{F}_{2}$-isoprostaness as an index of oxidative stress in vivo. Free Radic Biol Med $2000 ; 28$ : 505-13.

6. SIGNORINI C, COMPORTIM, GIORGI G. Ion trap tandem mass spectrometric determination of F2-isoprostanes. J Mass Spectrom 2003 ; 38 : 1067-74.

7. BERDEAUX O, SCRUEL O, DURAND T, CRACOWSKI I. Isoprostanes, biomarkers of lipid peroxidation in humans. Part 2: Quantification methods. Pathol Biol $2005 ; 53$ : 356-63.

8. GRECO A, MINGHETTI L. Isoprostanes as biomarkers and mediators pf oxidative injury in infant and adult central nervous system diseases. Curr Neurovasc Res $2004 ; 1$ : 341-54.

9. PROUDFOOT I, BARDENA, MORIT, et al. Measurement of urinary $F(2)$-isoprostanes as markers of in vivo lipid peroxidation-A comparison of enzyme immunoassay with gas chromatography/mass spectrometry. Anal Biochem $1999 ; 272$ : 209-15.

10. CHERUBINI A, RUGGIERO C, POLIDORIM, MECORI P. Potential markers of oxidative stress in stroke. Free Radic Biol Med 2005 ; 39 : 841 52.

11. ROBERT 2ND LI, FESSEL JP, DAVIES SS. The biochemistry of the isoprostane, neuroprostane, and isofuran pathways of lipid peroxidation. Brain Pathol 2005 ; 15 : 143-8.

12. BACOT S, BERNOUD-HUBAC N, BADDAS N, et al. Covalent binding of hydroxyl-alkenals 4-HDDE, 4-HHE, and 4-HNE to ethanolamine phospholipids classes. I Lipid Res 2003; 44 : 917-26.

13. GUICHARDANT $M$, CHANTEGREL B, DESHAYES C, DOUTHEAU A, MOLIĖRE P, LAGARDE M. Specific markers of lipid peroxidation issued from n-3 and n-6 fatty acids. Biochem Soc Trans 2004 ; 32 : 139-40. 